\title{
Irreversible Electroporation in Patients with Pancreatic Cancer: How Important Is the New Weapon?
}

\author{
Guo Tian, ${ }^{1,2}$ Xueping Liu, ${ }^{1,3}$ Qiyu Zhao, ${ }^{1}$ Danxia $X u^{1}{ }^{1}$ and Tian'an Jiang $\mathbb{D}^{1,2}$ \\ ${ }^{1}$ Department of Ultrasound Medicine, the First Affiliated Hospital, Zhejiang University School of Medicine, Hangzhou, China \\ ${ }^{2}$ Key Laboratory of Precision Diagnosis and Treatment for Hepatobiliary and Pancreatic Tumor of Zhejiang Province, \\ Hangzhou, China \\ ${ }^{3}$ Department of Ultrasound, People's Hospital, Quzhou, China
}

Correspondence should be addressed to Tian’an Jiang; tiananjiang@zju.edu.cn

Received 17 October 2017; Accepted 12 March 2018; Published 26 April 2018

Academic Editor: Helmut Schoellnast

Copyright (C) 2018 Guo Tian et al. This is an open access article distributed under the Creative Commons Attribution License, which permits unrestricted use, distribution, and reproduction in any medium, provided the original work is properly cited.

Background. Pancreatic cancer (PC) is a deadly disease with poor prognosis in the general population. We aimed to quantitate overall survival of patients with PC after irreversible electroporation (IRE) and the incidence of relevant complications. Methods. We performed a literature search via five electronic databases (PubMed, Embase, Web of Science, Scopus, and Cochrane Library databases) up to August 2017. The primary outcomes were overall survival and prognosis. Secondary outcomes included the response of post-IRE complications. Fixed-effects or random-effects meta-analysis was conducted to pool these data. Results. A total of 15 eligible articles involving 535 patients were included. The primary outcomes showed that the pooled prevalence estimates of overall survival were $94.1 \%$ (95\% CI: 90.7-97.5), 80.9\% (95\% CI: 72.5-89.4), 54.5\% (95\% CI: 38.3-70.6), and 33.8\% (95\% CI: $14.2-53.5)$ at $3,6,12$, and 24 months, and the pooled prevalence data of complete response (CR) at 2 months, partial response (PR) at 3 months, and progression at 3 months were 12.5\% (95\% CI: 2.9-22.2), 48.5\% (95\% CI: 39.4-57.6), and 19.7\% (95\% CI: 7.3-32.2), respectively. The secondary outcomes showed that the pooled prevalence values of post-IRE complications were abscess $6.6 \%(95 \%$ CI: $0.2-13$ ), fistula $10.6 \%$ (95\% CI: 2.5-18.7), pain 33.5\% (95\% CI: 14.5-52.5), infection 16.1\% (95\% CI: 3.9-28.4), thrombosis 4.9\% (95\% CI: 1.2-8.5), pancreatitis 7.2\% (95\% CI: 3.1-11.2), bleeding 4.2\% (95\% CI: -0.5-8.9), cholangitis 4.2\% (95\% CI: -0.5-8.9), nausea 9.6\% (95\% CI: 4.4-14.8), biliary obstruction $13.8 \%$ (95\% CI: 4.2-23.3), chest tightness 7.6\% (95\% CI: 0.5-14.6), and hypoglycemia $5.9 \%$ (95\% CI: -0.4-12.2). Conclusions. This meta-analysis indicated a clear survival benefit for PC patients who received irreversible electroporation therapy, although future safety and effectivity monitoring from more large-scale studies will be needed.

\section{Introduction}

Pancreatic cancer is a highly malignant disease with poor prognosis, which accounts for about $4 \%$ of cancer-related deaths in general population $[1,2]$. Although surgical resection provides chance for curable treatment, about only twenty percent of patients were resectable [3]. In recent decades, interventional therapies such as high-intensity focused ultrasound (HIFU) [4, 5], radiofrequency ablation (RFA) [6, 7], and microwave ablation (MWA) [8] have become fine ways in the treatment of pancreatic tumors.

Unlike those methods, the emerging family member in ablative techniques is irreversible electroporation (IRE), which is an invasive nonthermal ablation method by utilizing short direct current pulses to increase cell membrane permeability and result in cellular death [9]. Animal model showed that IRE produced the clear borders between treated and untreated area [10]. It would not be affected by the heat sink and suitable for lesions in the intricate regions. In recent decades, this technique has been generally reported in substantial number of tumor tissues located in liver $[11,12]$, pancreas $[13,14]$, lung [15], and kidney [16, 17], where IRE seemed to be effective. However, existing clinical experiences and sample size were still insufficient. In this regard, thus we performed the systematic review to estimate the efficacy and safety of IRE treatment for patients with PC in terms of overall survival and the complications. 


\section{Methods}

2.1. Search Strategy and Study Eligibility. The systematic review was conducted based on the Preferred Reporting Items for Systematic Reviews and Meta-Analyses (PRISMA) guidelines [18]. We cautiously did a literature search through five electronic databases (PubMed, Embase, Web of science, Scopus, and Cochrane Library databases) from inception to August 2017. Subject headings and keywords of irreversible electroporation and pancreatic cancer were retrieved (Supplementary Text). The retrieved references were managed using Endnote X7, and duplicates were filtered through the software.

2.2. Inclusion Criteria. Articles would be included when they met the following criteria: (1) they were published original reports; (2) they focused on IRE treatments of patients with PC; (3) they reported the overall survival, prognosis, or the IRE-related complications; and (4) follow-up interval was at least 1 month after IRE therapies. Literatures searched had no language restrictions. Systematic reviews, meta-analyses, conference presentations, and letters were excluded. All disagreements of opinion in this study were discussed and resolved by consensus with the third arbiter.

2.3. Data Extraction and Quality Assessment. At first, two individuals independently classified all literatures using the established protocols. The initial classification was evaluated by the third reviewer to maximize its accuracy. Then full-text papers were cross-checked by two investigators for further analysis according to inclusion criteria. Finally, two authors independently extracted the data from included studies using predefined protocols, such as author, study period, design style, country, population characteristics, tumor size, treatment methods, patients, gender, age, follow-up interval, complication, imaging methods, and prognosis. The primary outcomes were overall survival at 3-, 6-, 12- and 24-month time points and complete response at 2 months, partial response at 3 months, and progression at 3 months, and secondary outcomes included the incidence of post-IRE complications (abscess, fistula, pain, infection, thrombosis, pancreatitis, bleeding, cholangitis, nausea, biliary obstruction, chest tightness, and hypoglycemia).

We rated the quality of each subgroup as high, moderate, low, or very low based on the Grading of Recommendations Assessment, Development, and Evaluation (GRADE) guideline. Randomized controlled trials had an initial high evidence quality and were downgraded if present in the risk of bias, inconsistency, indirectness, imprecision, or publication bias, whereas observational studies began with a low evidence quality and may be upgraded in terms of large effect, plausible confounding, or dose-response gradient [19].

The pooled incidence rates of overall survival and complications were calculated to combine the summary result from each subgroup. $I^{2}$ statistics, chi-square test $\left(\chi^{2}\right)$, and $\tau^{2}$ were used for the assessment of statistical between-study heterogeneity. $p$ value of 0.05 or less indicated statistical significance. If $I^{2}$ values less than $50 \%$ were considered little heterogeneity, then a fixed-effect model was used. Otherwise, a random-effects model was performed for substantial heterogeneity [20]. In this study, sensitivity analysis was to check the impact of each study on the whole incidence assessment by consecutively deleting each study. Bias to small study effects was quantitatively estimated by the Egger test [21]. All analysis in this study was performed using Stata 12.0 software.

\section{Results}

3.1. Study Characteristics. A total of 789 articles were identified through those five databases, of which 774 records were duplicates and removed based on predefined inclusion criteria. Finally, a total of 15 studies related to IRE and PC were included (Figure 1) [9, 13, 22-34]. These studies involved 535 cases (female $46.5 \%$ ), in which the mean age of participants was ranging from 53 to 69 years, and there were 8 studies conducted in Europe, 4 in Asia, and 3 from North America. These were all described in Table 1 . The risk of methodological quality bias for all outcomes in most studies ranged from low to moderate levels due to their limited sample size, selection bias, and publication bias (Supplementary Table 1).

3.2. Primary and Secondary Outcomes. As shown in Supplementary Table 1 and Figures 2 and 3, the primary outcomes showed that the pooled prevalence estimates of overall survival were 94.1\% (95\% CI: 90.7-97.5), 80.9\% (95\% CI: 72.5-89.4), 54.5\% (95\% CI: 38.3-70.6), and 33.8\% (95\% CI: $14.2-53.5)$ at $3,6,12$, and 24 months, with obvious evidence of between-study heterogeneity $\left(I^{2}=60.9 \%\right.$ and $p=0.002$; $I^{2}=79.2 \%$ and $p<0.001 ; I^{2}=91.9 \%$ and $p<0.001$; $I^{2}=89.3 \%$ and $\left.p<0.001\right)$. And the pooled prevalence data of $\mathrm{CR}$ at 2 months, $\mathrm{PR}$ at 3 months, progression at 3 months were $12.5 \%$ (95\% CI: 2.9-22.2), 48.5\% (95\% CI: 39.4-57.6), and 19.7\% (95\% CI: 7.3-32.2) (Supplementary Figures 1-12), in which the heterogeneity between studies was acceptable except CR $\left(I^{2}=0\right.$ and $p=0.793 ; I^{2}=42.5 \%$ and $p=0.122$; $I^{2}=77.8 \%$ and $\left.p<0.001\right)$. Furthermore, among 4 studies, there were detectable publication bias stratified by the overall survival through Egger test $(t=-5.77$ and $p<0.001$; $t=-5.28$ and $p<0.001 ; t=-2.43$ and $p=0.041 ; t=-24.73$ and $p=0.026$ )

The secondary outcomes showed that the pooled incidence values of post-IRE complications were abscess $6.6 \%$ (95\% CI: $0.2-13$ ), fistula $10.6 \%$ (95\% CI: $2.5-18.7$ ), pain $33.5 \%$ (95\% CI: 14.5-52.5), infection 16.1\% (95\% CI: 3.9-28.4), thrombosis $4.9 \%$ (95\% CI: $1.2-8.5)$, pancreatitis $7.2 \%(95 \%$ CI: 3.1-11.2), bleeding 4.2\% (95\% CI: $-0.5-8.9$ ), cholangitis 4.2\% (95\% CI: $-0.5-8.9$ ), nausea 9.6\% (95\% CI: 4.4-14.8), biliary obstruction $13.8 \%$ (95\% CI: 4.2-23.3), chest tightness 7.6\% (95\% CI: 0.5-14.6), and hypoglycemia 5.9\% (95\% CI: -0.4-12.2), in which between-study heterogeneity was found in pain subgroup $\left(I^{2}=74.8 \%\right.$ and $\left.p=0.008\right)$. During the publication bias assessment in post-IRE complications, it showed that nausea and hypoglycemia were significant $(t=$ 7.63 and $p=0.005 ; t=34.22$ and $p=0.019)$.

Additionally, sensitivity analysis stratified by primary and secondary outcomes indicated that there was no individual 


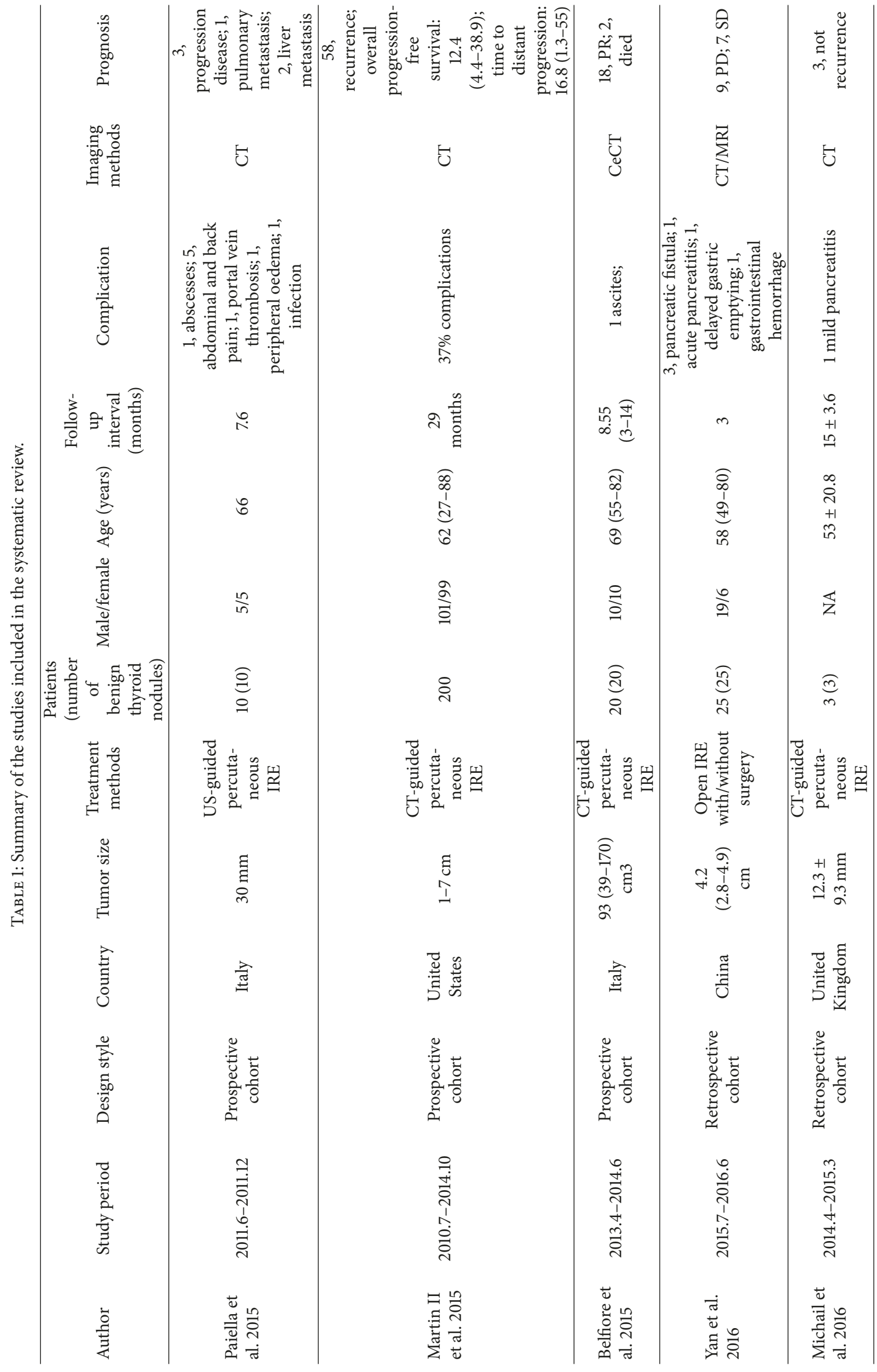




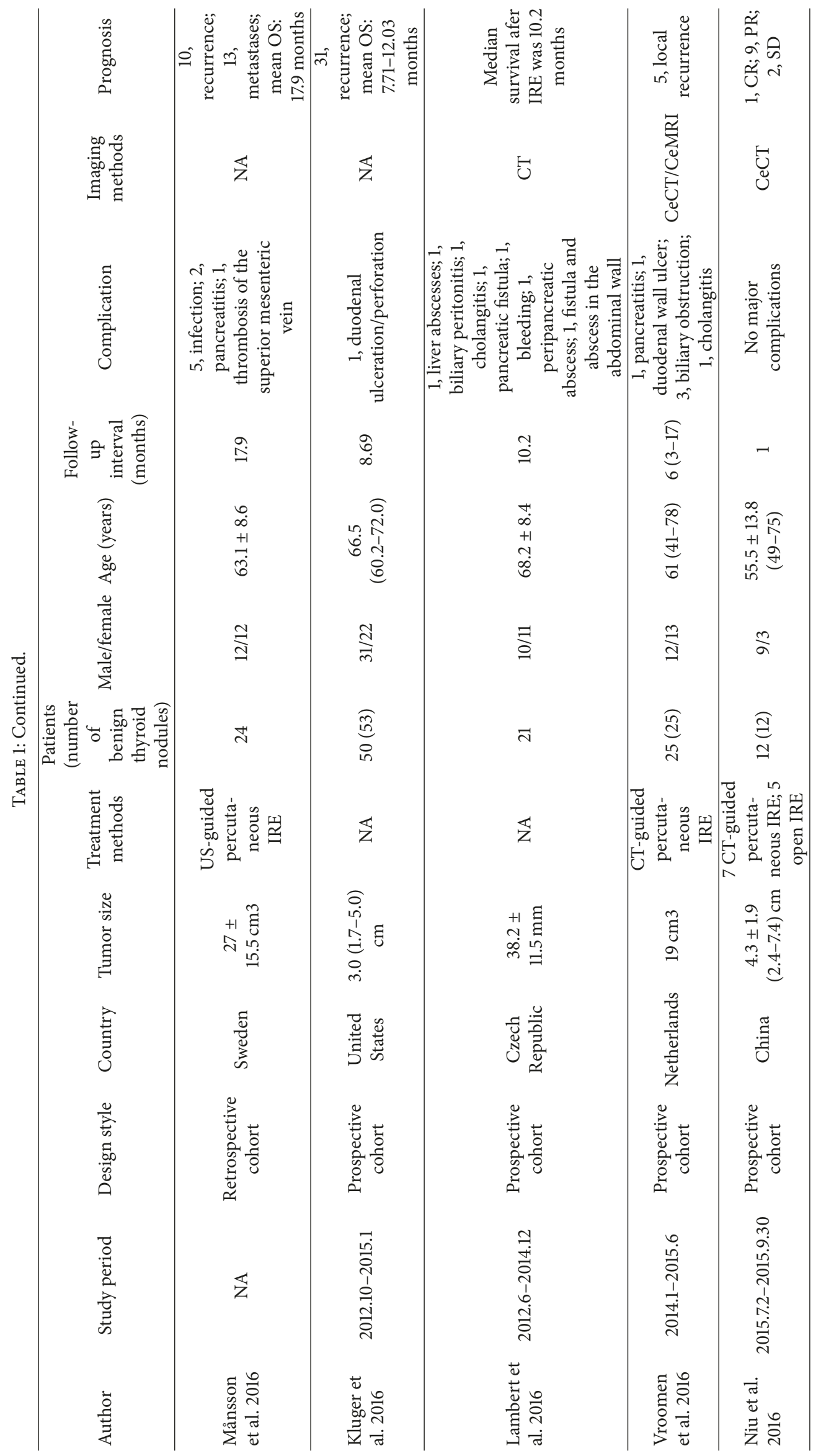




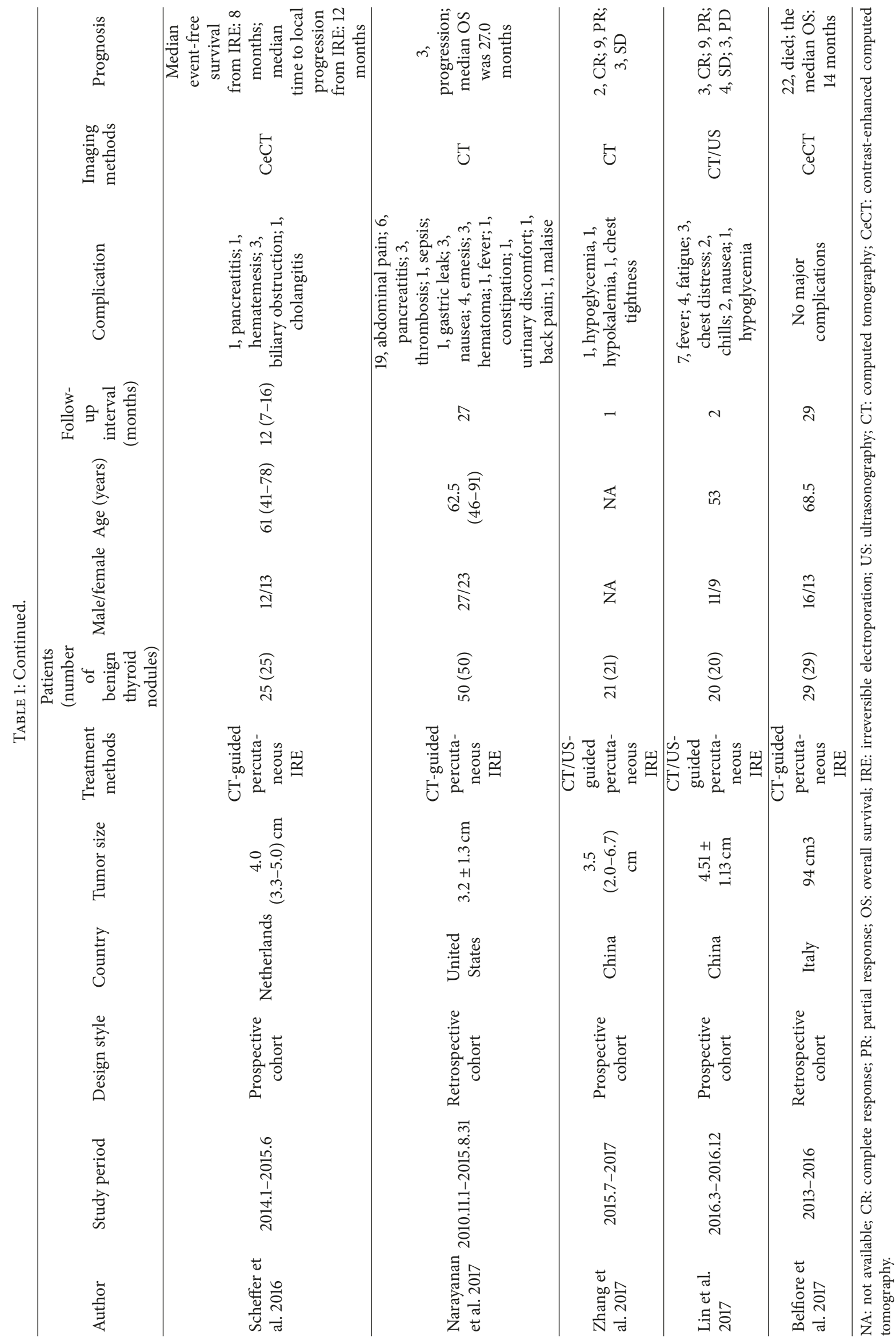




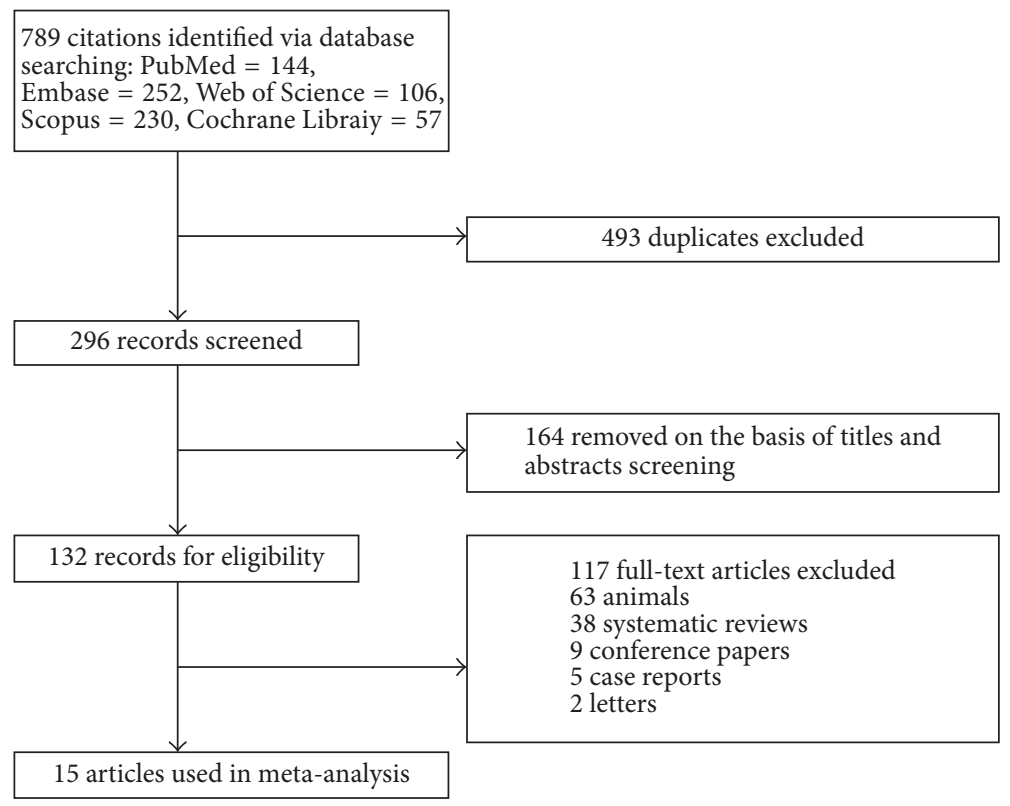

Figure 1: Study selection process.

article significantly changing the overall prevalence estimate, by repeating meta-analysis after deleting each study.

\section{Discussion}

This systematic review is the most comprehensive evaluation of clinical benefits in IRE treatment of patients with PC. Regarding the outcome of IRE, we carried out 15 separate articles and found that overall survival values were $94.1 \%$, $80.9 \%, 54.5 \%$, and $33.8 \%$ at $3,6,9,12$, and 24 months, and the pooled incidence data of CR at 2 month, PR at 3 month, progression at 3 month were $12.5 \%, 48.5 \%$, and $19.7 \%$, respectively. A low mortality in IRE could result from main gastrointestinal surgery and other serious diseases in these patients [26]. However, if RFA were applied in PC, the results showed complication rates (15\%) and a progressionfree survival rate of $22 \%$ during the follow-up of 12 months [35]. Previous studies showed that the proportion of 7-month survival in PC patients in resection and no resection group was $53.19 \%$ versus $70.40 \%$ [36], and the 12 -month overall survival in the radiation and nonradiation group was $43 \%$ and $29 \%$, respectively [37]. In fact, as an emerging technique, IRE was usually conducted percutaneously or by open surgical or laparoscopic access, which was superior in unresectable lesions. In addition, it was usually used if the lesions were not suitable for other thermal ablations such as MWA, RFA, and cryoablation due to the proximity to sensitive structures such as important blood vessels and gastrointestinal tract. IRE may induce cell apoptosis in the case of supporting the intact cellular structure, and thus PC treatment was enabled, and it was well-known that the pancreas was deep seated in the retroperitoneum. In short, our study showed that IRE had been favorable in prolonging survival, and IRE of PC could be safer than other thermal ablation procedures [38].
Another interesting finding from this study was that postIRE complications remained a crucial concern during the process of IRE for pancreatic cancer. It was reported that the rate of postpercutaneous IRE complications ranged from 0 to $37 \%[9,27,31]$, most of which were shown self-limiting and curable with relatively low incidence, such as abscess (7\%), nausea (10\%), chest tightness (8\%), and hypoglycemia (6\%). In RFA for PC, postprocedural adverse events occurred in pancreatic fistula (4\%-18.8\%), gastrointestinal hemorrhage $(4 \%-18.8 \%)$, thrombosis $(4.7 \%-15.4 \%)$, and acute pancreatitis $(2 \%-11.5 \%)[35,38-40]$. It was reported that some complications like bowel and biliary perforation in RFA and MWA could be directly induced by thermal injury [41]. Previous study showed that IRE increased heating to cause "white zone" thermal coagulation in case of high energy intensity, which should be not negligible and may be related to thermal damage inducing cell death, confirmed by a thick rim of Hsp70, a marker of thermal damage adjacent to the zone of ablation, similar to thermal ablations [42]. It may be that this thermal damage would be advantageous to facilitate the effect of IRE therapy via enlarging the treatment scope. In addition, it was advised that temperature monitoring could be in consideration during the procedure of IRE ablation around vital structures [43]. This finding would be crucial to further study, based on the low rates of complications reported by IRE.

The present study has several limitations explained as follows. First, relatively small samples could partially account for the main heterogeneity. Particularly in primary outcomes, it may result from the variation of limited participants number in each study. Second, historical treatments and individual differences in studies may have effect on the present heterogeneity. Third, this analysis did not differentiate patients for both early and late stages of pancreatic cancer and their percentage of position distribution, which could 


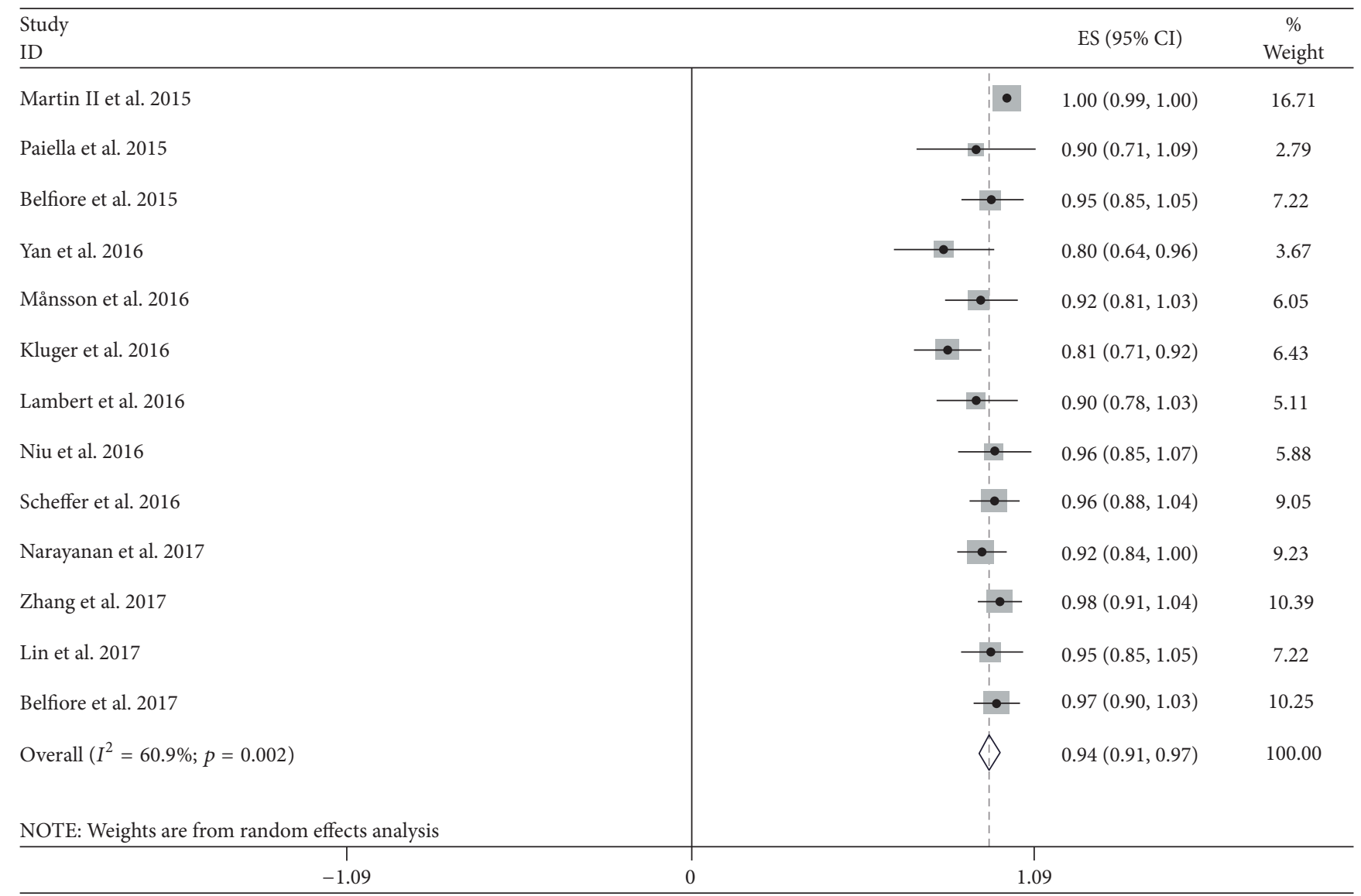

(a)

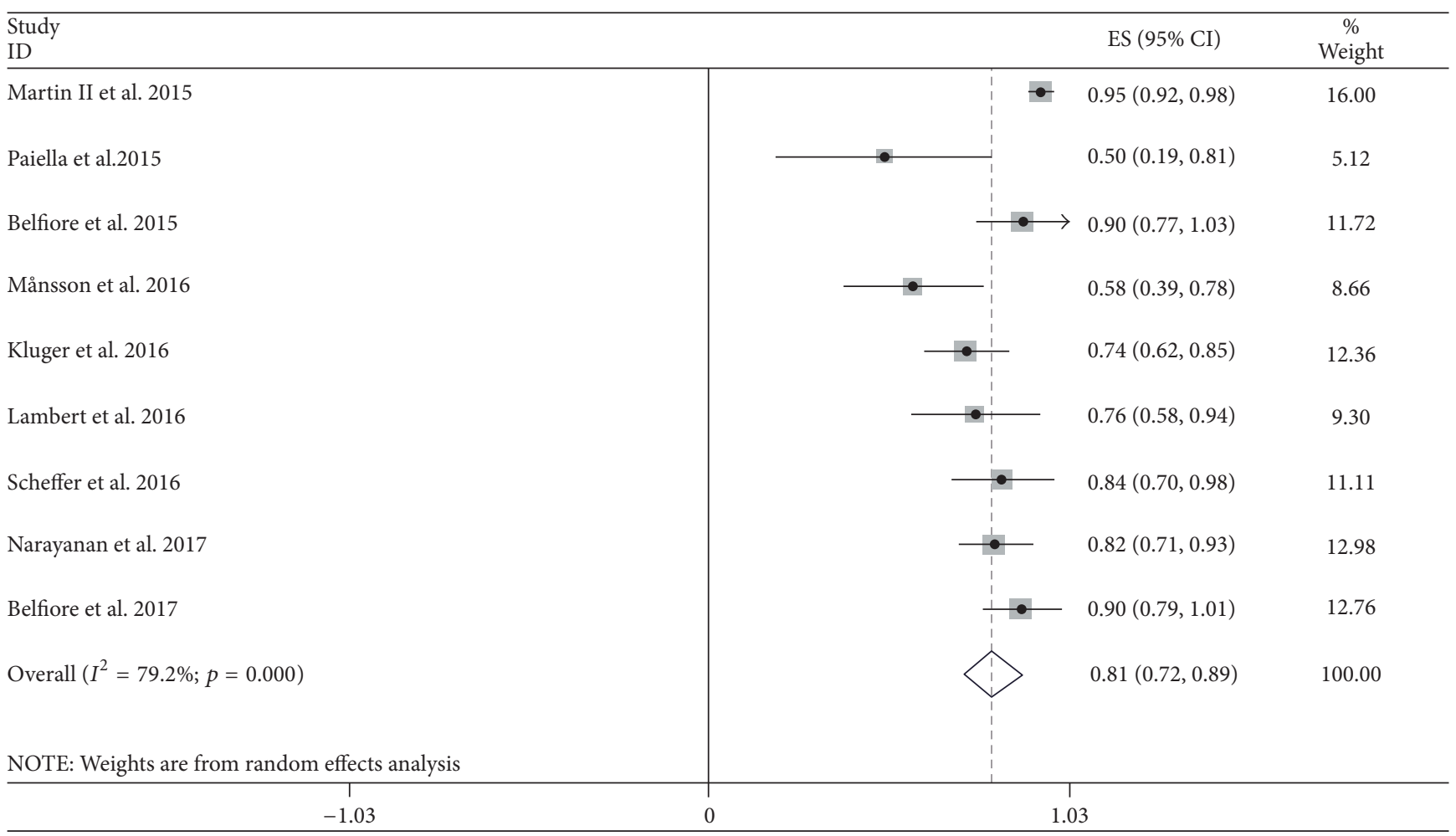

(b)

Figure 2: Continued. 


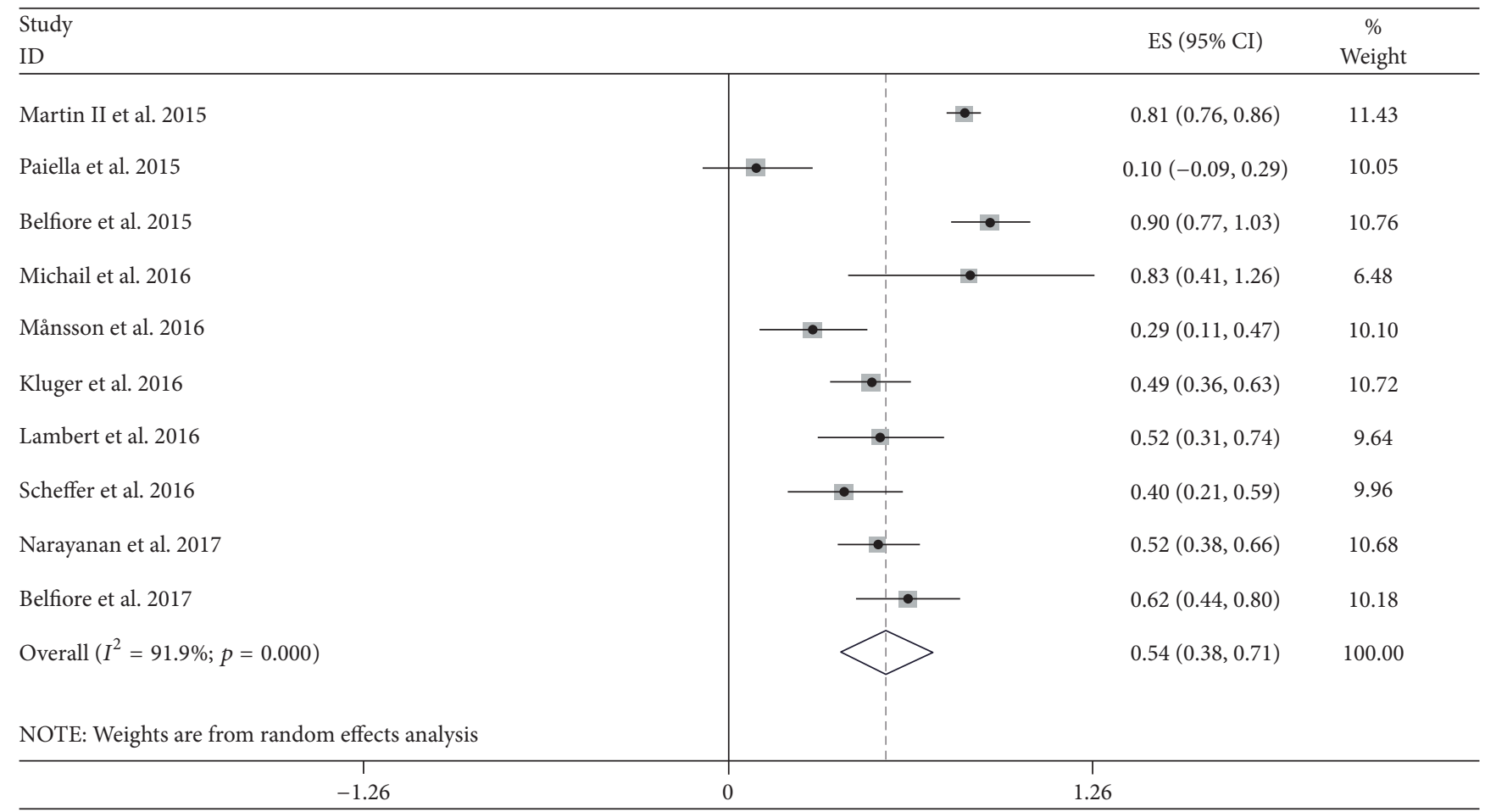

(c)

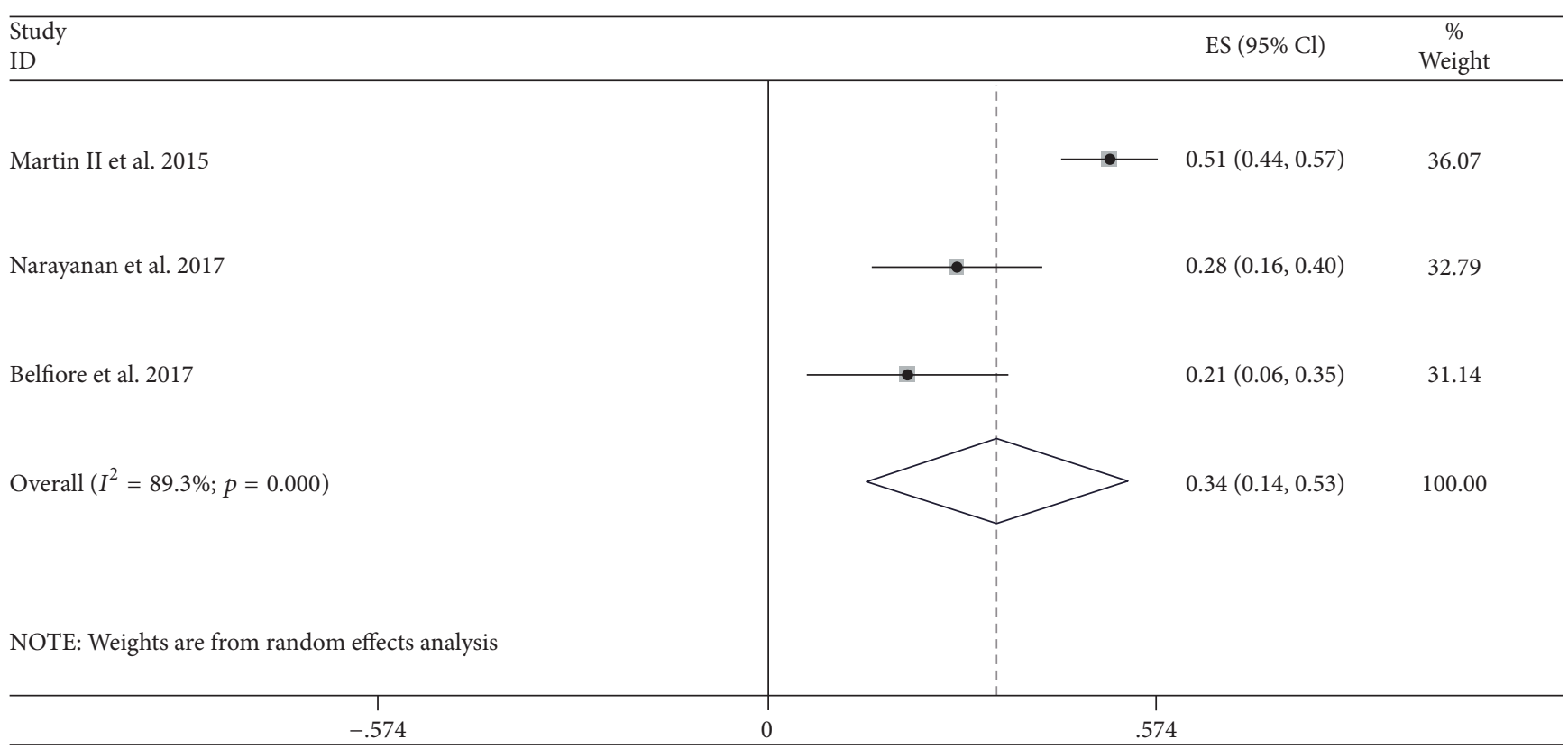

(d)

FIGURE 2: Forest plot of overall survival among studies targeting the effect of IRE therapy in patients with PC at 3 (a), 6 (b), 12 (c), and 24 (d) months.

be involved in this study as confounding factors, but we did not have enough power to perform subgroup analysis. Fourth, the assessment risk of evidence quality using GRADE criteria was subjective, and tests for publication bias should be seriously interpreted given that the results may be overestimated.

\section{Conclusions}

In summary, though evidence of IRE-related injury in the pancreas was relatively low, the potential for damage needed further consideration. This meta-analysis indicated that IRE may be safe and effective in prolonging survival for patients 


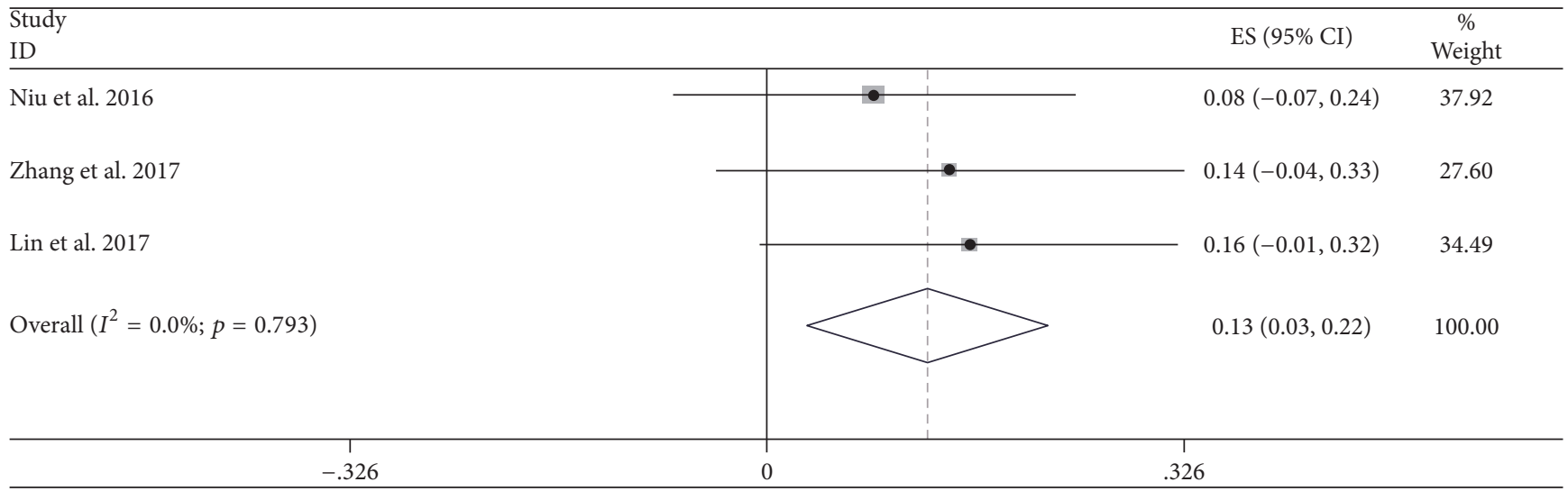

(a)

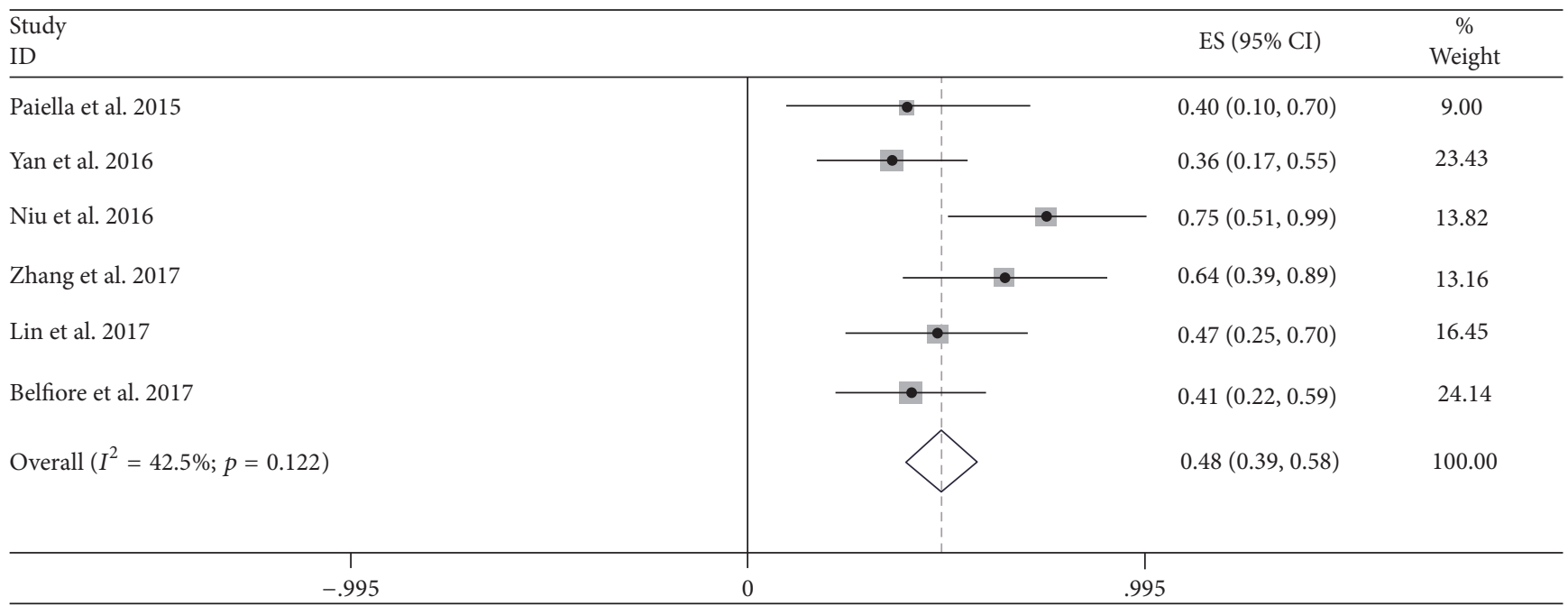

(b)

\begin{tabular}{|c|c|c|c|}
\hline $\begin{array}{l}\text { Study } \\
\text { ID }\end{array}$ & & ES $(95 \%$ CI) & $\begin{array}{c}\% \\
\text { Weight }\end{array}$ \\
\hline Belfiore et al. 2015 & $\longrightarrow$ & $0.10(-0.03,0.23)$ & 18.52 \\
\hline Paiella et al. 2015 & & $0.30(0.02,0.58)$ & 10.54 \\
\hline Yan et al. 2016 & $\longrightarrow$ & $0.16(0.02,0.30)$ & 17.81 \\
\hline Månsson et al. 2016 & & $0.54(0.34,0.74)$ & 14.61 \\
\hline Narayanan et al. 2017 & $\bullet$ & $0.06(-0.01,0.13)$ & 21.91 \\
\hline Lin et al. 2017 & $\bullet$ & $0.16(-0.01,0.32)$ & 16.62 \\
\hline Overall $\left(I^{2}=77.8 \% ; p=0.000\right)$ & & $0.20(0.07,0.32)$ & 100.00 \\
\hline NOTE: Weights are from random effect & & & \\
\hline $\begin{array}{r} \\
-.741 \\
\end{array}$ & 0 & 41 & \\
\hline
\end{tabular}

(c)

FIGURE 3: Forest plot of the pooled prevalence data of complete response at 2 months (a), partial response at 3 months (b), and progression at 3 months (c) among studies targeting the effect of IRE therapy in patients with PC. 
with pancreatic cancers. More pairwise-comparison studies estimating IRE against traditional surgical resection and interventional therapies will be needed to identify that clinical benefits are available.

\section{Abbreviations in Order of Appearance}

\author{
PC: $\quad$ Pancreatic cancer \\ IRE: Irreversible electroporation \\ CR: $\quad$ Complete response \\ PR: $\quad$ Partial response \\ PRISMA: Preferred Reporting Items for Systematic \\ Reviews and Meta-Analyses \\ HIFU: High-intensity focused ultrasound \\ RFA: Radiofrequency ablation \\ MWA: Microwave ablation \\ GRADE: Grading of Recommendations \\ Assessment, Development, and \\ Evaluation.
}

\section{Conflicts of Interest}

The authors state that there are no conflicts of interest regarding the publication of this paper.

\section{Authors' Contributions}

(1) Study concept and design were by Guo Tian. (2) Acquisition of data was conducted by Guo Tian, Xueping Liu, Qiyu Zhao, and Danxia Xu. (3) Analysis and interpretation of data were performed by Guo Tian and Qiyu Zhao. (4) Drafting of the manuscript was by Guo Tian. (5) Critical revision of the manuscript for important intellectual content was done by Tian'an Jiang. (6) Statistical analysis was conducted by Guo Tian, Xueping Liu, Qiyu Zhao, and Danxia Xu. (7) Funding was obtained by Qiyu Zhao and Tian'an Jiang. (8) Technical or material support was provided by Tian'an Jiang. (9) Study supervision was by Tian'an Jiang.

\section{Acknowledgments}

This study was supported by the Natural Science Foundation of Zhejiang Province (LY16H180004); the Science and Technology Project of Zhejiang Province (2012C23094); and the Foundation of Zhejiang Health Committee (2014KYA086).

\section{Supplementary Materials}

Supplementary Text 1: search strategy. Supplementary Table 1: subgroup analysis and quality assessment of included studies by primary outcomes and secondary outcomes. Supplementary Figure 1: forest plot of the incidence rate ratios of abscess after IRE therapy for patients with PC. Supplementary Figure 2: forest plot of the incidence rate ratios of fistula after IRE therapy for patients with PC. Supplementary Figure 3: forest plot of the incidence rate ratios of pain after IRE therapy for patients with PC. Supplementary Figure 4: forest plot of the incidence rate ratios of infection after IRE therapy for patients with PC. Supplementary Figure 5: forest plot of the incidence rate ratios of thrombosis after IRE therapy for patients with PC. Supplementary Figure 6: forest plot of the incidence rate ratios of pancreatitis after IRE therapy for patients with PC. Supplementary Figure 7: forest plot of the incidence rate ratios of bleeding after IRE therapy for patients with PC. Supplementary Figure 8: forest plot of the incidence rate ratios of cholangitis after IRE therapy for patients with PC. Supplementary Figure 9: forest plot of the incidence rate ratios of nausea after IRE therapy for patients with PC. Supplementary Figure 10: forest plot of the incidence rate ratios of biliary obstruction after IRE therapy for patients with PC. Supplementary Figure 11: forest plot of the incidence rate ratios of chest tightness after IRE therapy for patients with PC. Supplementary Figure 12: forest plot of the incidence rate ratios of hypoglycemia after IRE therapy for patients with PC. (Supplementary Materials)

\section{References}

[1] C. Are and A. Patel, "Current status and future directions in the management of pancreatic cancer," Indian Journal of Surgical Oncology, vol. 6, no. 1, pp. 3-5, 2015.

[2] A. Carrato, A. Falcone, M. Ducreux et al., "A systematic review of the burden of pancreatic cancer in Europe: real-world impact on survival, quality of life and costs," Journal of Gastrointestinal Cancer, vol. 46, no. 3, pp. 201-211, 2015.

[3] S. J. Rombouts, J. A. Vogel, H. C. van Santvoort et al., "Systematic review of innovative ablative therapies for the treatment of locally advanced pancreatic cancer," British Journal of Surgery, vol. 18, no. 3, p. e748, 2016.

[4] J. Zhao, F. Zhao, Y. Shi, Y. Deng, X. Hu, and H. Shen, “The efficacy of a new high intensity focused ultrasound therapy for locally advanced pancreatic cancer," Journal of Cancer Research and Clinical Oncology, vol. 143, no. 10, pp. 2105-2111, 2017.

[5] M. Marinova, M. Rauch, M. Mücke et al., "High-intensity focused ultrasound (HIFU) for pancreatic carcinoma: evaluation of feasibility, reduction of tumour volume and pain intensity," European Radiology, vol. 26, no. 11, pp. 4047-4056, 2016.

[6] M. D’Onofrio, S. Crosara, R. De Robertis et al., "Percutaneous radiofrequency ablation of unresectable locally advanced pancreatic cancer: preliminary results," Technology in Cancer Research \& Treatment, vol. 16, no. 3, pp. 285-294, 2017.

[7] S. Varshney, A. Sewkani, S. Sharma et al., "Radiofrequency ablation of unresectable pancreatic carcinoma: feasibility, efficacy and safety," Journal of the Pancreas, vol. 7, no. 1, pp. 74-78, 2006.

[8] G. Carrafiello, A. M. Ierardi, F. Fontana et al., "Microwave ablation of pancreatic head cancer: safety and efficacy," Journal of Vascular and Interventional Radiology, vol. 24, no. 10, pp. 1513-1520, 2013.

[9] R. C. Martin II, D. Kwon, S. Chalikonda et al., “Treatment of 200 locally advanced (stage III) pancreatic adenocarcinoma patients with irreversible electroporation: safety and efficacy," Annals of Surgery, vol. 262, no. 3, pp. 486-494, 2015.

[10] J. F. Edd, L. Horowitz, R. V. Davalos, L. M. Mir, and B. Rubinsky, "In vivo results of a new focal tissue ablation technique: irreversible electroporation," IEEE Transactions on Biomedical Engineering, vol. 53, no. 7, pp. 1409-1415, 2006.

[11] W. Cheung, H. Kavnoudias, S. Roberts, B. Szkandera, W. Kemp, and K. R. Thomson, "Irreversible electroporation for 
unresectable hepatocellular carcinoma: initial experience and review of safety and outcomes," Technology in Cancer Research \& Treatment, vol. 12, no. 3, pp. 233-241, 2013.

[12] K. R. Thomson, W. Cheung, S. J. Ellis et al., "Investigation of the safety of irreversible electroporation in humans," Journal of Vascular and Interventional Radiology, vol. 22, no. 5, pp. 611-621, 2011.

[13] S. Paiella, G. Butturini, and I. Frigerio, "Safety and feasibility of Irreversible Electroporation (IRE) in patients with locally advanced pancreatic cancer: results of a prospective study," Digestive Surgery, vol. 32, no. 2, pp. 90-97, 2015.

[14] D. Kwon, K. McFarland, V. Velanovich, and R. C. G. Martin II, "Borderline and locally advanced pancreatic adenocarcinoma margin accentuation with intraoperative irreversible electroporation," Surgery, vol. 156, no. 4, pp. 910-922, 2014.

[15] M. Usman, W. Moore, R. Talati, K. Watkins, and T. V. Bilfinger, "Irreversible electroporation of lung neoplasm: a case series," Medical Science Monitor, vol. 18, no. 6, pp. CS43-CS47, 2012.

[16] J. J. Wendler, J. Ricke, M. Pech et al., "First delayed resection findings after irreversible electroporation (IRE) of human localised renal cell carcinoma (RCC) in the IRENE pilot phase 2a trial," CardioVascular and Interventional Radiology, vol. 39, no. 2, pp. 239-250, 2016.

[17] M. Pech, A. Janitzky, J. J. Wendler et al., "Irreversible electroporation of renal cell carcinoma: a first-in-man phase I clinical study," Cardio Vascular and Interventional Radiology, vol. 34, no. 1, pp. 132-138, 2011.

[18] A. Liberati, D. G. Altman, J. Tetzlaff et al., "The PRISMA statement for reporting systematic reviews and meta-analyses of studies that evaluate health care interventions: explanation and elaboration," Journal of Clinical Epidemiology, vol. 62, no. 10, pp. el-e34, 2009.

[19] H. Balshem, M. Helfand, and H. J. Schunemann, "GRADE guidelines: rating the quality of evidence-introduction," Journal of Clinical Epidemiology, vol. 64, pp. 4401-406, 2011.

[20] H. Jpt and S. Green, "Cochrane handbook for systematic reviews of interventions version 5.1.0," The Cochrane Database of Systematic Reviews, vol. 2011, no. 2, p. S38, 2011.

[21] J. A. C. Sterne and M. Egger, "Funnel plots for detecting bias in meta-analysis: guidelines on choice of axis," Journal of Clinical Epidemiology, vol. 54, no. 10, pp. 1046-1055, 2001.

[22] M. P. Belfiore, F. M. Ronza, F. Romano et al., "Percutaneous CTguided irreversible electroporation followed by chemotherapy as a novel neoadjuvant protocol in locally advanced pancreatic cancer: our preliminary experience," International Journal of Surgery, vol. 21, supplement 1, pp. S34-S39, 2015.

[23] L. Yan, Y.-L. Chen, M. Su et al., "A single-institution experience with open irreversible electroporation for locally advanced pancreatic carcinoma," Chinese Medical Journal, vol. 129, no. 24, pp. 2920-2925, 2016.

[24] P. Michail, A. Ali, M. Pizanias, P. Peddu, J. Karani, and N. Heaton, "Irreversible electroporation for the treatment of pancreatic neuroendocrine tumors," Korean Journal of HepatoBiliary-Pancreatic Surgery, vol. 20, no. 3, pp. 116-120, 2016.

[25] C. Månsson, R. Brahmstaedt, A. Nilsson, P. Nygren, and B.-M. Karlson, "Percutaneous irreversible electroporation for treatment of locally advanced pancreatic cancer following chemotherapy or radiochemotherapy," European Journal of Surgical Oncology, vol. 42, no. 9, pp. 1401-1406, 2016.

[26] M. D. Kluger, I. Epelboym, B. A. Schrope et al., "Singleinstitution experience with irreversible electroporation for T4 pancreatic cancer: first 50 patients," Annals of Surgical Oncology, vol. 23, no. 5, pp. 1736-1743, 2016.

[27] H. Scheffer, L. Vroomen, B. Zonderhuis et al., "Ablation of locally advanced pancreatic carcinoma by percutaneous irreversible electroporation: results of the phase I/II PANFIREstudy," Radiology, vol. 18, no. 2, Article ID 152835, p. e115, 2016.

[28] L. G. P. H. Vroomen, H. J. Scheffer, M. C. A. M. Melenhorst et al., "MR and CT imaging characteristics and ablation zone volumetry of locally advanced pancreatic cancer treated with irreversible electroporation," European Radiology, vol. 27, no. 6, pp. 2521-2531, 2016.

[29] L. Z. Niu, J. Y. Zeng, and Y. S. Zhang, "Irreversible electroporation ablation therapy for pancreatic adenocarcinoma: observation of its safety and short-term effect," Journal of Vascular and Interventional Radiology, vol. 25, no. 3, pp. 225230, 2016.

[30] L. Lambert, J. Horejs, Z. Krska et al., "Treatment of locally advanced pancreatic cancer by percutaneous and intraoperative irreversible electroporation: general hospital cancer center experience," Neoplasma, vol. 63, no. 2, pp. 269-273, 2016.

[31] G. Narayanan, P. J. Hosein, I. C. Beulaygue et al., "Percutaneous image-guided irreversible electroporation for the treatment of unresectable, locally advanced pancreatic adenocarcinoma," Journal of Vascular and Interventional Radiology, vol. 28, no. 3, pp. 342-348, 2017.

[32] Y. Zhang, J. Shi, J. Zeng et al., "Percutaneous irreversible electroporation for ablation of locally advanced pancreatic cancer experience from a Chinese institution," Pancreas, vol. 46, no. 2, pp. e12-e14, 2017.

[33] M. Lin, S. Liang, X. Wang et al., "Short-term clinical efficacy of percutaneous irreversible electroporation combined with allogeneic natural killer cell for treating metastatic pancreatic cancer," Immunology Letters, vol. 186, pp. 20-27, 2017.

[34] G. Belfiore, M. P. Belfiore, A. Reginelli et al., "Concurrent chemotherapy alone versus irreversible electroporation followed by chemotherapy on survival in patients with locally advanced pancreatic cancer," Medical Oncology, vol. 34, no. 3, article 38, 2017.

[35] R. Girelli, I. Frigerio, A. Giardino et al., "Results of 100 pancreatic radiofrequency ablations in the context of a multimodal strategy for stage III ductal adenocarcinoma," Langenbeck's Archives of Surgery, vol. 398, no. 1, pp. 63-69, 2013.

[36] S. Walczak and V. Velanovich, "An evaluation of artificial neural networks in predicting pancreatic cancer survival," Journal of Gastrointestinal Surgery, vol. 152, no. 5, p. S1248, 2017.

[37] M. Sajjad, S. Batra, S. Hoffe, R. Kim, G. Springett, and A. Mahipal, "Use of radiation therapy in locally advanced pancreatic cancer improves survival: a SEER database analysis," American Journal of Clinical Oncology, 2016.

[38] R. Girelli, I. Frigerio, R. Salvia, E. Barbi, P. Tinazzi Martini, and C. Bassi, "Feasibility and safety of radiofrequency ablation for locally advanced pancreatic cancer," British Journal of Surgery, vol. 97, no. 2, pp. 220-225, 2010.

[39] G. Boz, A. De Paoli, R. Innocente et al., "A triple approach strategy for patients with locally advanced pancreatic adenocarcinoma," International Journal of Radiation Oncology Biology Physics, vol. 81, no. 2, pp. 623-627, 2011.

[40] S. John, D. Anastasios, M. Nikolaos et al., "High operative risk of cool-tip radiofrequency ablation for unresectable pancreatic head cancer," Journal of Surgical Oncology, vol. 96, no. 1, pp. 8990, 2007. 
[41] M. Akahane, H. Koga, N. Kato et al., "Complications of percutaneous radiofrequency ablation for hepatocellular carcinoma: imaging spectrum and management," RadioGraphics, vol. 25, supplement 1, pp. S57-S68, 2005.

[42] M. Faroja, M. Ahmed, L. Appelbaum et al., "Irreversible electroporation ablation: is all the damage nonthermal?" Radiology, vol. 266, no. 2, pp. 462-470, 2013.

[43] P. G. Wagstaff, D. M. de Bruin, W. van den Bos et al., "Irreversible electroporation of the porcine kidney: temperature development and distribution," Urologic Oncology: Seminars and Original Investigations, vol. 33, no. 4, pp. 168.el-168.e7, 2015. 


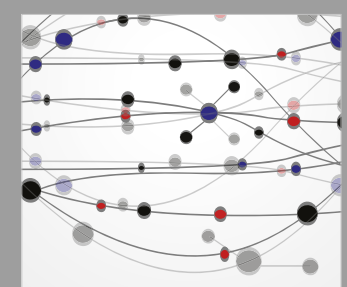

The Scientific World Journal
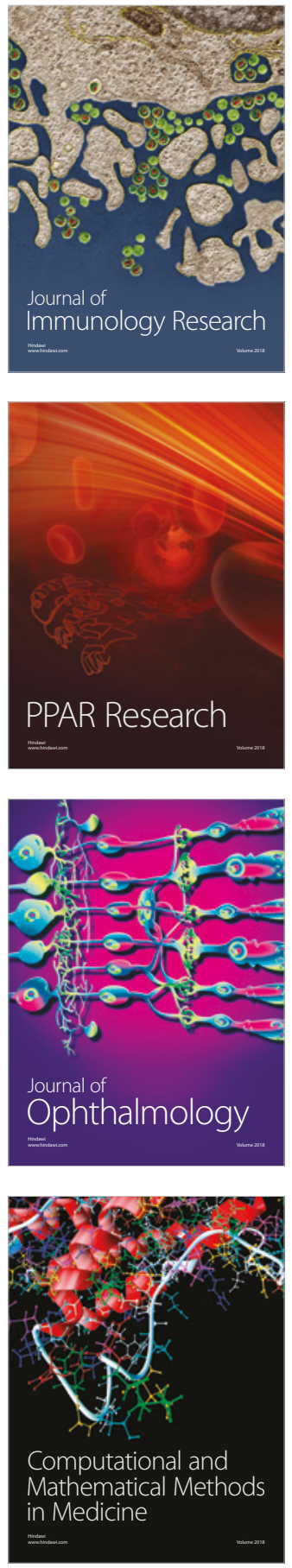

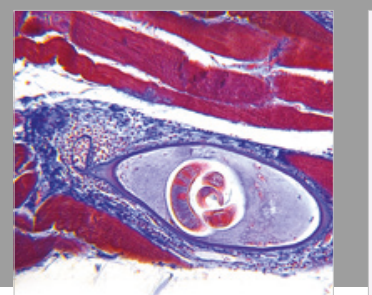

Gastroenterology Research and Practice

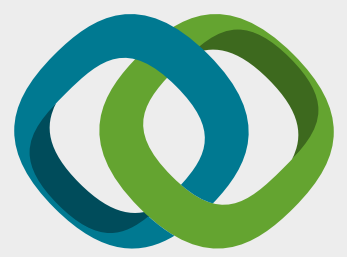

\section{Hindawi}

Submit your manuscripts at

www.hindawi.com
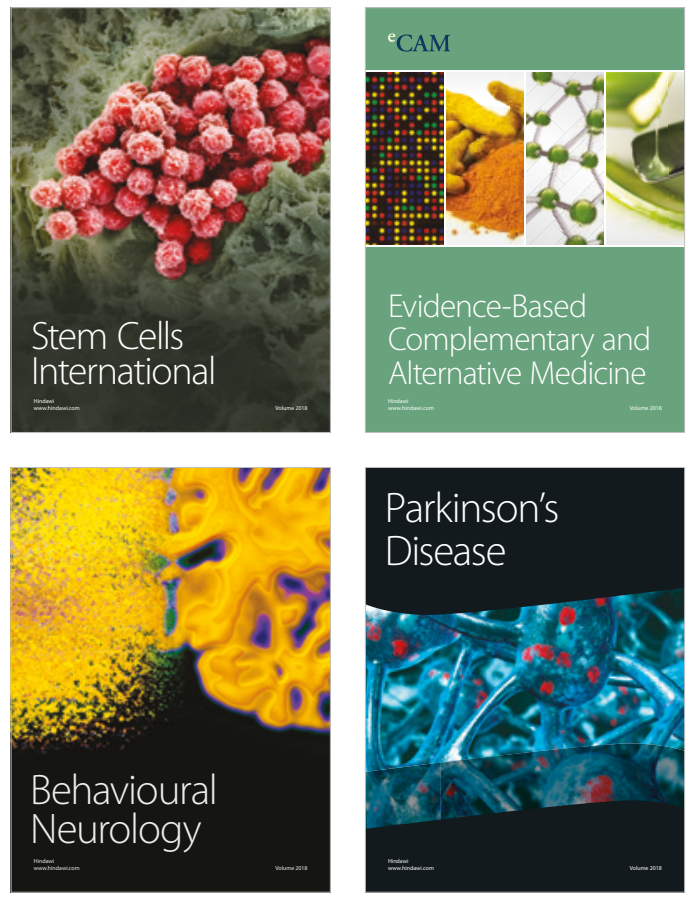

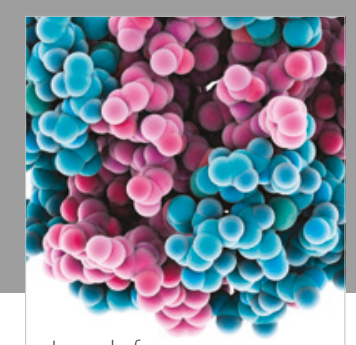

Journal of

Diabetes Research
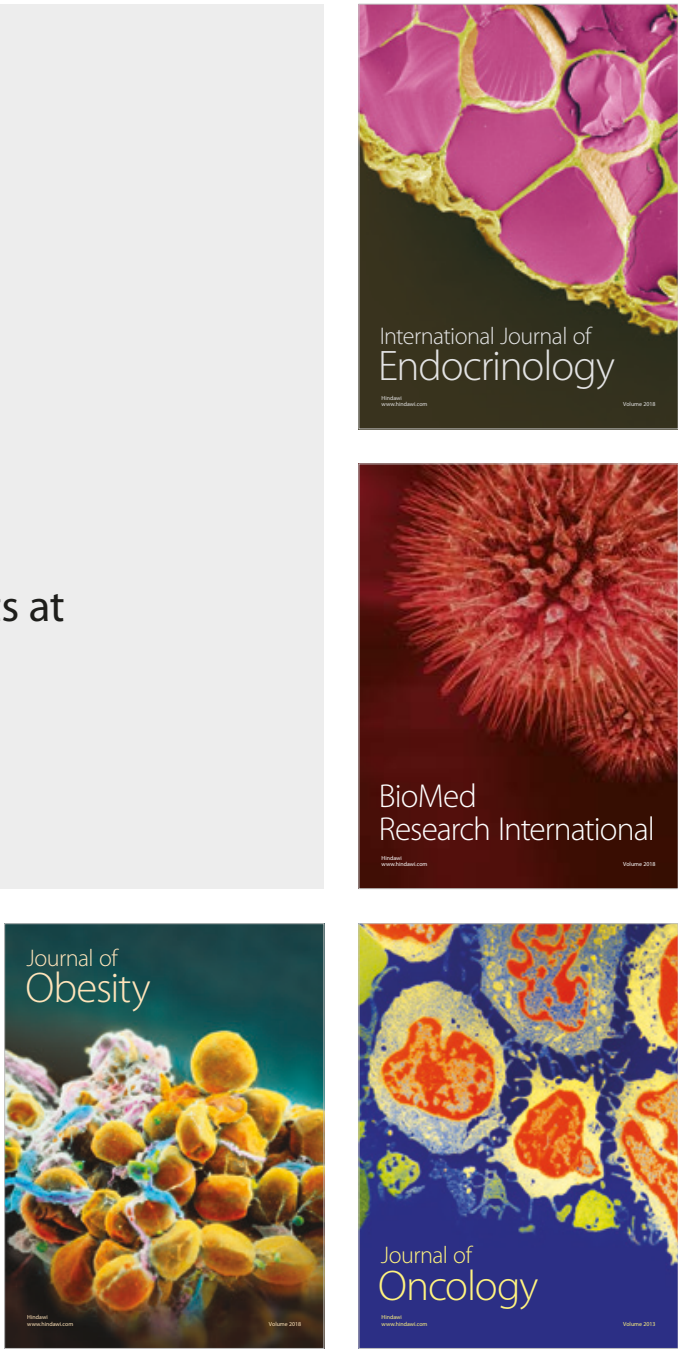

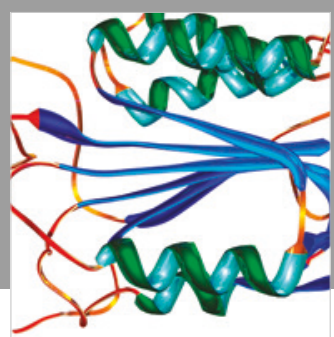

Disease Markers
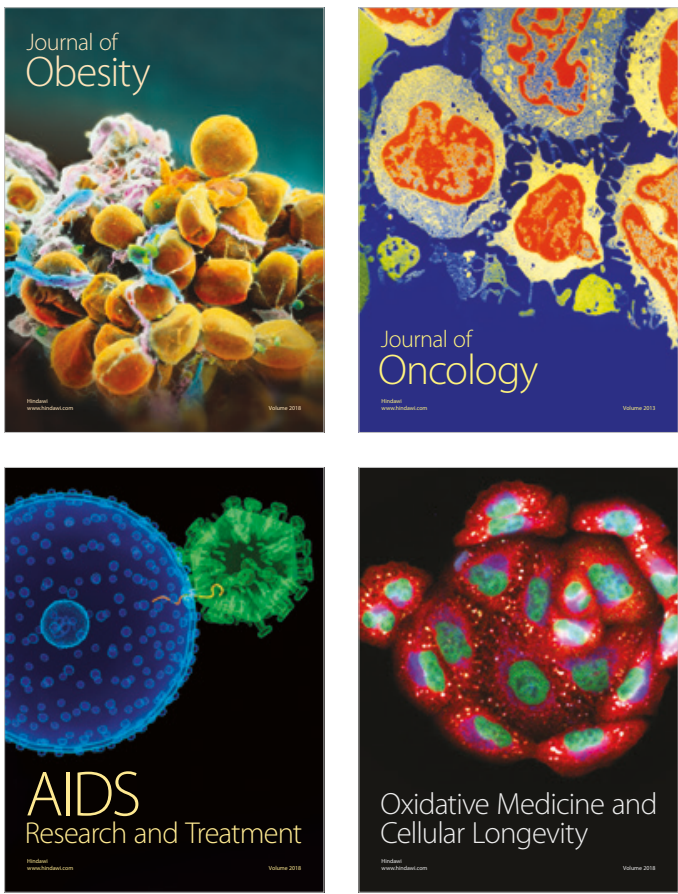